\title{
SHOCK WAVES AND COSMIC RAY ACCELERATION IN THE OUTSKIRTS OF GALAXY CLUSTERS
}

\author{
Sungwook E. Hong ${ }^{1,2}$, Dongsu Ryu ${ }^{1}$, Hyesung Kang ${ }^{3}$, and Renyue Cen ${ }^{4,5}$ \\ ${ }^{1}$ Department of Astronomy and Space Science, Chungnam National University, Daejeon 305-764, Korea; swhong@canopus.cnu.ac.kr, ryu@canopus.cnu.ac.kr \\ 2 Department of Physics, Korea Institute for Advanced Study, Seoul 130-722, Korea \\ ${ }^{3}$ Department of Earth Sciences, Pusan National University, Busan 609-735, Korea; hskang@pusan.ac.kr \\ ${ }^{4}$ Department of Astrophysical Sciences, Princeton University, NJ 08544, USA; cen@ astro.princeton.edu \\ Received 2013 September 7; accepted 2014 March 3; published 2014 April 3
}

\begin{abstract}
The outskirts of galaxy clusters are continuously disturbed by mergers and gas infall along filaments, which in turn induce turbulent flow motions and shock waves. We examine the properties of shocks that form within $r_{200}$ in sample galaxy clusters from structure formation simulations. While most of these shocks are weak and inefficient accelerators of cosmic rays (CRs), there are a number of strong, energetic shocks which can produce large amounts of CR protons via diffusive shock acceleration. We show that the energetic shocks reside mostly in the outskirts and a substantial fraction of them are induced by infall of the warm-hot intergalactic medium from filaments. As a result, the radial profile of the CR pressure in the intracluster medium is expected to be broad, dropping off more slowly than that of the gas pressure, and might be even temporarily inverted, peaking in the outskirts. The volume-integrated momentum spectrum of CR protons inside $r_{200}$ has the power-law slope of 4.25-4.5, indicating that the average Mach number of the shocks of main CR production is in the range of $\left\langle M_{s}\right\rangle_{\mathrm{CR}} \approx 3-4$. We suggest that some radio relics with relatively flat radio spectrum could be explained by primary electrons accelerated by energetic infall shocks with $M_{s} \gtrsim 3$ induced in the cluster outskirts.
\end{abstract}

Key words: acceleration of particles - cosmic rays - galaxies: clusters: intracluster medium - methods: numerical - shock waves

Online-only material: color figures

\section{INTRODUCTION}

Clusters of galaxies are the largest gravitationally bound structures that emerged from hierarchical clustering during the large-scale structure (LSS) formation of the universe. While the central part of many clusters looks relaxed into hydrostatic equilibrium, especially in X-ray observations (e.g., Markevitch et al. 1998; Vikhlinin et al. 2006), the outskirts around the virial radius, $r_{\text {vir }}$, are stirred by mergers of substructures and continuous infall of gas along adjacent filaments (e.g., Ryu et al. 2003; Skillman et al. 2008; Vazza et al. 2009a). Observational evidence for the deviation from equilibrium in the cluster outskirts can be seen in the entropy distribution. The radial profile of $S \equiv k T / n_{e}^{2 / 3}$ obtained in X-ray observations follows roughly $\sim r^{1.1}$ in the inner part of clusters, but beyond it $S$ flattens off and turns down (Voit et al. 2005; George et al 2009; Walker et al. 2012; Simionescu et al. 2013). Moreover, according to structure formation simulations, turbulent flow motions develop during the formation of clusters; the ratio of turbulence to gas pressure increases outward and reaches of the order of $10 \%$ in the outskirts of simulated clusters (e.g., Ryu et al. 2008; Vazza et al. 2009b; Lau et al. 2009).

Although flow motions are expected to be on average subsonic in the cluster outskirts, ${ }^{6}$ shock waves have been observed in X-ray as well as in radio. In X-ray observations, some of sharp discontinuities in the surface brightness are attributed to shocks, while others are attributed to cold fronts or contact discontinuities. The physical properties of these shocks including the sonic Mach number, $M_{s}$, can be determined using the deprojected temperature and density jumps (Markevitch \& Vikhlinin

\footnotetext{
5 Author to whom all correspondence should be addressed.

6 The ratio of turbulent to gas pressure of $\sim 30 \%$, for instance, corresponds to the turbulent Mach number of $\sim 0.42$.
}

2007). Since a shock was found in the so-called bullet cluster (1E 065756; Markevitch et al. 2002), about a dozen of shocks have been detected with Chandra, XMM-Newton, and recently Suzaku (e.g., Russell et al. 2010; Akamatsu et al. 2012; Ogrean $\&$ Brüggen 2013). The shocks identified so far in X-ray observations are mostly weak with $M_{s} \sim 1.5-3$.

Shocks have been identified also in radio observations through the so-called radio relics (see, e.g., Feretti et al. 2012; Brüggen et al. 2012 for reviews). Radio relics are the radio structures of megaparsec size observed within the virial radius. They often show elongated morphologies with sharp edges in one side, and occasionally come in pairs located in opposite sides of clusters. Radio emissions from these structures usually exhibit high polarization fractions. Radio relics are interpreted as shocks, where relativistic electrons emitting synchrotron radiation are accelerated or re-accelerated. The properties of radio relic shocks such as $M_{s}$, magnetic field strength, and the age can be estimated from the spectral index and spatial profile of synchrotron emissions (van Weeren et al. 2010; Kang et al. 2012). So far several dozens of radio relics have been observed, and the Mach numbers of associated shocks are typically in the range of $M_{s} \sim 1.5-4.5$ (e.g., Clarke \& Enßlin 2006; Bonafede et al. 2009; van Weeren et al. 2010, 2012).

In a few cases, shocks were detected both in X-ray and radio observations. Interestingly, however, the shock characteristics derived from X-ray observations are not always consistent with those from radio observations. For instance, the shock in the so-called sausage relic in CIZA J2242.8+5301 was estimated to have $M_{s} \simeq 4.6$ in the analysis of radio spectrum based on diffusive shock acceleration (DSA) model (van Weeren et al. 2010), but X-ray observations indicated $M_{s} \simeq 3.2$ (Akamatsu \& Kawahara 2013). And the shock in the so-called toothbrush relic in 1RXS J0603.3+4214 has $M_{s} \simeq 3.3-4.6$ according to the radio spectral analysis, but $M_{s} \lesssim 2$ according to X-ray observations 
(van Weeren et al. 2012; Ogrean et al. 2013). In addition, the positions of shocks identified in radio are often spatially shifted from those found in X-ray (see the references above). Resolving these puzzles would require further observations as well as theoretical understandings of weak collisionless shocks in the intracluster medium (ICM), which is a high beta plasma with $\beta=P_{\text {th }} / P_{B} \sim 100$ (e.g., Ryu et al. 2008). Here, $P_{\text {th }}$ and $P_{B}$ are the gas thermal and magnetic pressures, respectively.

With relatively low Mach numbers as well as elongated morphologies and occasional parings in opposite sides of clusters, shocks observed in the outskirts are often considered to be induced by mergers. The hypothesis of merger shocks was explored in simulated clusters, especially for the origin of radio relics (Skillman et al. 2011, 2013; Nuza et al. 2012). In these studies, shocks in clusters are identified and the injection and acceleration of cosmic-ray (CR) electrons are modeled. Then, along with a model for the magnetic field in the intergalactic medium (IGM), synthetic radio maps are produced and examined. These studies suggested that merger shocks with sufficient kinetic energy flux are likely to be responsible for observed radio relics. However, it was also argued that typical mergers are expected to induce mostly weak shocks with $M_{s} \lesssim 3$ and major mergers with similar masses, which are required to explain, for instance, the sausage relic (van Weeren et al. 2010), tend to generate very weak shocks with $M_{s} \lesssim 2$ (e.g., Gabici \& Blasi 2003).

The nature and origin of cosmological shocks have been studied extensively, using numerical simulations for the LSS formation of the universe (Minati et al. 2000; Ryu et al. 2003; Pfrommer et al. 2006; Kang et al. 2007; Skillman et al. 2008; Hoeft et al. 2008; Vazza et al. 2009a; Brüggen et al. 2012). Shocks are induced as a consequence of hierarchical clustering of nonlinear structures and can be classified into two categories. External shocks form around clusters and filaments of galaxies, when the cool $\left(T \sim 10^{4} \mathrm{~K}\right)$, tenuous gas in voids accretes onto them. So the Mach number of external shocks can be very high, reaching up to $M_{s} \sim 100$ or so. Internal shocks, which form inside nonlinear structures, on the other hand, have lower Mach numbers of $M_{s} \lesssim 10$ or so, because they form in much hotter gas that was previously shocked. It was shown that while a large fraction of internal shocks have $M_{s} \lesssim 3$, those with $2 \lesssim M_{s} \lesssim 4$ are most important in dissipating the shock kinetic energy into heat in the ICM. Internal shocks are induced by mergers of substructures, as well as by turbulent flow motions and by infall of warm gas from filaments to clusters (e.g., Ryu et al. 2003).

Turbulent shocks, induced by turbulent flow motions, are expect to be weak with at most $M_{s} \lesssim 2$, because the rms flow motions are subsonic. Inflall shocks, ${ }^{7}$ on the contrary, can have Mach numbers as large as $\sim 10$, since they form by the infall of the so-called warm-hot intergalactic medium (WHIM) with $T \approx 10^{5}-10^{7} \mathrm{~K}$ to the hot ICM with $T \approx 10^{7}-10^{8} \mathrm{~K}$. We note that a continuous infall containing density clumps would be difficult to be differentiated from a stream of minor mergers with small mass ratios. However, infall shocks clearly differ from merger shocks, which are generated by major mergers, in the sense that they do not appear as a pair in opposite sides of clusters. In addition, infall shocks should be found mostly in the cluster outskirts, since the gas infall from filaments normally stops around the virial radius and does not penetrate into the

\footnotetext{
7 Here we distinguish infall shocks from external accretion shocks that decelerate never-shocked gas accreting onto clusters and filaments from void regions. Infall shocks are by nature also accretion shocks that stop previously shocked gas accreting onto clusters from filaments.
}

core. So it would be reasonable to conjecture that while weak shocks with $M_{s} \lesssim 3$ in clusters are mostly merger or turbulent shocks, stronger shocks with $M_{s} \gtrsim 3$ found in the cluster outskirts are likely to be infall shocks.

Shocks that can be categorized as infall shocks were identified in observations before. For instance, the radio relic $1253+275$ in the Coma cluster was interpreted as an infall shock formed by a group of galaxies along with the intra-group medium accreting into the ICM (Brown \& Rudnick 2011). Also the radio structure of NGC 1265 in the Perseus Cluster was modeled as the passage of the galaxy through a shock with $M_{s} \simeq 4.2$ formed by the infalling WHIM (Pfrommer \& Jones 2011). However, the properties such as the frequency, spatial distribution, and energetics of infall shocks have not been studied in simulations before, partly because the automated distinction of infall shocks from merger shocks or other types of shocks in simulated clusters is not trivial.

It is well established that CRs are produced via DSA process at collisionless shocks, such as interplanetary shocks, supernova remnant shocks, and shocks in clusters (Bell 1978; Blandford \& Ostriker 1978; Drury 1983). Shocks in the LSS of the universe are the primary means through which the gravitational energy released during the structure formation is dissipated into the gas entropy, turbulence, magnetic field, and CR particles (e.g., Ryu et al. 2008, 2012). Post-processing estimations with simulation data for the amount of CR protons produced in clusters showed that the CR pressure in the ICM may reach up to a few percent of the gas thermal pressure (Ryu et al. 2003; Kang et al. 2007; Skillman et al. 2008; Vazza et al. 2009a). Observationally, on the other hand, the CR-to-thermal pressure ratio in clusters was constrained to be less than a few percent, with the upper limits on $\gamma$-ray fluxes set by Fermi-LAT and VERITAS (Ackermann et al. 2010, 2013; Arlen et al. 2012).

In some simulations for the LSS formation, the injection/ acceleration of $\mathrm{CR}$ protons at shocks and the spatial advection of the CR pressure were followed self-consistently in runtime (e.g., Miniati et al. 2001; Pfrommer et al. 2007; Vazza et al. 2012). Pfrommer et al. (2007) and Vazza et al. (2012), adopting specific DSA efficiency models, showed that the CR acceleration occurs mostly in the cluster outskirts. Because of long lifetime and slow particle diffusion (e.g., Berezinsky et al. 1997), the CR protons accelerated in the outskirts are likely to be contained in clusters and accumulated in the ICM over cosmological timescales. But they can be advected with turbulent flows toward the central part of the cluster (see, e.g., Enßlin et al. 2011). For simplicity, let us assume that the transport of $\mathrm{CR}$ protons due to flow motions can be approximated by turbulent diffusion, then it could be described by $\partial Q(\boldsymbol{r}, t) / \partial t=\nabla \cdot[D(\boldsymbol{r}, t) \nabla Q(\boldsymbol{r}, t)]$, where $Q(\boldsymbol{r}, t)$ is the density of CR protons and $D(\boldsymbol{r}, t)$ is the turbulent diffusion coefficient. If only the radial diffusion is considered and the diffusion coefficient is approximated as $D(r, t) \sim r V(r)$, where $V(r)$ is the average flow speed at $r$, then the advection timescale can be estimated rather roughly as $\tau_{\text {adv }} \sim r^{2} / D \sim r / V(r)$. In the cluster outskirts, typically $r \sim 1 h^{-1} \mathrm{Mpc}$ and $V(r) \sim$ a few $\times 100 \mathrm{~km} \mathrm{~s}^{-1}$, so $\tau_{\text {adv }} \sim$ a few $\times 10^{9} \mathrm{yr}$. This is a substantial fraction of the age of the universe, implying that it would take a while for CR protons produced at energetic shocks in the outskirts to reach the core region. As a result, the radial distribution of the CR pressure would be broad, dropping off more slowly than that of the gas thermal pressure in the outskirts. Vazza et al. (2012) also showed that the CR pressure distribution could be temporarily inverted, that is, the CR pressure can increase outward. 
Brunetti et al. (2012), on the other hand, attempted to constrain the radial distributions of nonthermal components (including the CR proton energy density) in the Coma cluster by combining radio observations with recent Fermi-LAT $\gamma$-ray observations and with Faraday rotation measure (RM) data. They argued that the model based on the turbulent acceleration of secondary electrons would best reproduce the radio halo of the Coma cluster with the CR energy density that scales with the thermal energy density as $\varepsilon_{\mathrm{CR}} \propto \varepsilon_{\text {th }}^{\theta}$ with $\theta \approx-0.1$ to -0.35 , implying that $\varepsilon_{\mathrm{CR}}$ is higher at lower $\varepsilon_{\mathrm{th}}$. The outer region of the Coma cluster is strongly disturbed by ongoing mergers and infalls (e.g., Simionescu et al. 2013), so it would be probably one of rare cases with this kind of inversion of the $\varepsilon_{\mathrm{CR}}$ profile. But these indicate that the partitioning of thermal and CR energies (and possibly turbulent and magnetic field energies too) could be very different in different parts of clusters.

In this paper, we study shocks within the virial radius in a sample of clusters taken from LSS formation simulations. Specifically, we examine the properties of energetic shocks with relatively high Mach number and high shock kinetic energy flux that can produce large amounts of CR protons via DSA. The plan of this paper is as follows. In Section 2, numerical details are presented. In Section 3, the properties and nature of shocks in the cluster outskirts are described. In Section 4, the properties of CRs produced at energetic shocks are described. Discussion is given in Section 5, and summary follows in Section 6.

\section{NUMERICAL DETAILS}

\subsection{Cluster Sample}

To produce a sample of galaxy clusters, we performed simulations of the LSS formation, using a particle-mesh/Eulerian cosmological hydrodynamics code described in Ryu et al. (1993). A standard $\Lambda \mathrm{CDM}$ cosmological model was assumed with the following parameters: baryon density $\Omega_{\mathrm{BM}}=0.044$, dark matter density $\Omega_{\mathrm{DM}}=0.236$, cosmological constant $\Omega_{\Lambda}=0.72$, Hubble parameter $h \equiv H_{0} /\left(100 \mathrm{kms}^{-1} \mathrm{Mpc}^{-1}\right)=0.7$, rms density fluctuation $\sigma_{8}=0.82$, and primordial spectral index $n=0.96$. These parameters are consistent with the WMAP7 data (Komatsu et al. 2011). Cubic boxes of comoving sizes of 100 and $200 h^{-1} \mathrm{Mpc}$ with periodic boundaries were employed and divided into $1024^{3}$ grid zones with uniform spatial resolutions of $\Delta l=97.7$ and $195.3 h^{-1} \mathrm{kpc}$, respectively. Nongravitational processes such as radiative cooling, star formation and feedback, and reionization of the IGM were not considered. Instead, a temperature floor was set to be $T_{\min }=10^{4} \mathrm{~K}$ for the gas in voids, assuming that the unshocked gas outside nonlinear structures was uniformly heated by reionization. To compensate the cosmic variance and acquire an enough number of clusters, 16 runs with different realizations of initial condition were performed for each of 100 and $200 h^{-1} \mathrm{Mpc}$ boxes (so the total number of runs is 32 ).

In addition, we used a higher resolution simulation with $2048^{3}$ grid zones in box of $100 h^{-1}$ Mpc comoving size $(\Delta l=$ $48.8 h^{-1} \mathrm{kpc}$ ), to mainly examine the resolution effects. This simulation was performed with a numerical code described in $\mathrm{Li}$ et al. (2008), adopting the same set of cosmological parameters except $\Omega_{\mathrm{BM}}=0.046$. It is basically the same simulation reported in Cen \& Chisari (2011), but with the box size of $100 h^{-1} \mathrm{Mpc}$ instead of $50 \mathrm{~h}^{-1} \mathrm{Mpc}$. The simulation includes a mild feedback from star formation (low galactic superwind feedback of Cen \& Chisari 2011) and cooling/heating processes. Kang et al. (2007) examined the effects of a similar feedback and

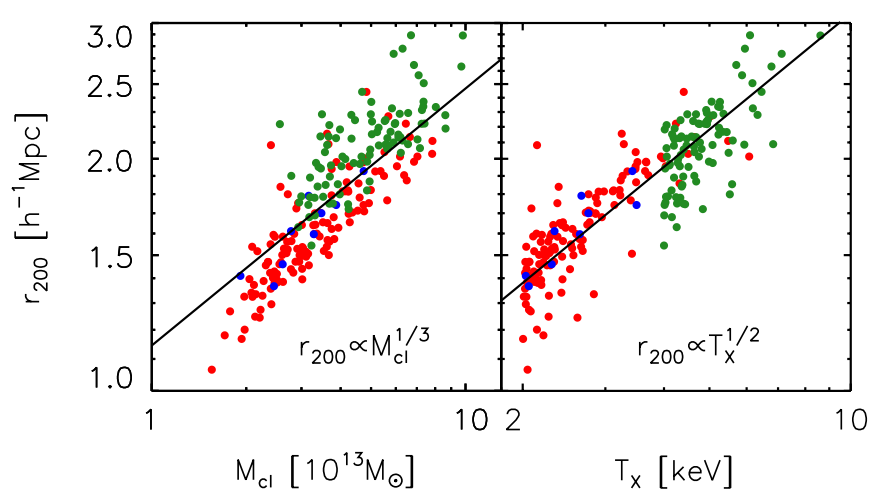

Figure 1. Radius as a function gas mass (left) and temperature (right) for clusters in our sample. Refer to the main text for definitions of $r_{200}, M_{\mathrm{cl}}$, and $T_{\mathrm{X}}$. Red dots denote 125 clusters with $T_{\mathrm{X}} \geqslant 2 \mathrm{keV}$ from $100 \mathrm{~h}^{-1} \mathrm{Mpc}$ box simulations with $1024^{3}$ grid zones, green dots denote 94 clusters with $T_{\mathrm{X}} \geqslant 4 \mathrm{keV}$ from $200 \mathrm{~h}^{-1} \mathrm{Mpc}$ box simulations with $1024^{3}$ zones, and blue dots denote 9 clusters with $T_{\mathrm{X}} \geqslant 2 \mathrm{keV}$ from $100 h^{-1} \mathrm{Mpc}$ box simulation with $2048^{3}$ zones, respectively. Solid lines represent the scaling relations among $r_{200}$, $M_{\mathrm{cl}}$, and $T_{\mathrm{X}}$, expected from virial equilibrium.

(A color version of this figure is available in the online journal.)

radiative processes on the properties of shocks in the LSS. They showed that the dynamics and energetics of shocks are governed primarily by the gravity of matters, so mild feedback and cooling do not significantly affect the statistics of the shocks in the ICM (see Pfrommer et al. 2007 for the case that the feedback is stronger and its effects are more important).

In the simulation data, we identified clusters as the volumes with high X-ray luminosity (see Kang et al. 1994 for details). For each identified cluster, we calculated the gas mass, $M_{\mathrm{cl}}$, and the X-ray emission-weighted average temperature, $T_{\mathrm{X}}$, inside $r_{200}$ from the cluster center that locates at the peak of X-ray emissivity. Here $r_{200}$ is defined as the radius within which the gas overdensity is 200 times the mean gas density (not the critical density) of the universe. ${ }^{8}$ We built our sample with clusters of $T_{\mathrm{X}} \geqslant 2 \mathrm{keV}$ from $100 \mathrm{~h}^{-1} \mathrm{Mpc}$ box simulations and those of $T_{\mathrm{X}} \geqslant 4 \mathrm{keV}$ from $200 \mathrm{~h}^{-1} \mathrm{Mpc}$ box simulations, by optimizing the resolution limitation and the size of cluster sample; 125 clusters were identified from 16 simulations of $100 h^{-1} \mathrm{Mpc}$ box with $1024^{3}$ zones, 94 clusters from 16 simulations of $200 h^{-1}$ Mpc box with $1024^{3}$ zones, and 9 clusters from one simulation of $100 h^{-1} \mathrm{Mpc}$ box with $2048^{3}$ zones. Figure 1 shows the radius-mass relation and the radius-temperature relation of the total 228 clusters in our sample. The simulated clusters have $r_{200} \approx 1-3 h^{-1} \mathrm{Mpc}, M_{\mathrm{cl}} \approx 10^{13}-10^{14} M_{\odot}$, and $T_{\mathrm{X}} \approx 2-10 \mathrm{keV}$. From the virial theorem, the mass, temperature, and radius of relaxed clusters are expected to follow $M_{\mathrm{vir}} \propto r_{\mathrm{vir}}^{3}$ and $T_{\text {vir }} \propto M_{\text {vir }} / r_{\text {vir }}$ or $r_{\text {vir }} \propto M_{\text {vir }}^{1 / 3} \propto T_{\text {vir }}^{1 / 2}$ (e.g., Peebles 1980). As can be seen from Figure 1 , overall $M_{\mathrm{cl}}, T_{\mathrm{X}}$, and $r_{200}$ of our clusters follow these relations, but there are scatters, a part of which are caused by dynamical activities in the cluster outskirts.

We point that with uniform grids of $\Delta l=48.8-195.3 h^{-1} \mathrm{kpc}$, our simulated clusters have poorer resolution than those generated using adaptive mesh refinement or smoothed particle hydrodynamics codes (see Introduction for references), especially in the core regions. This means that shocks in the inner regions of clusters may not be fully reproduced. However, those shocks in high-density regions are expected to be weak with low

\footnotetext{
8 The relation between $r_{200}$ and $r_{\mathrm{vir}}$ is rather complicated and depends on cosmological parameters (e.g., Nakamura \& Suto 1997; Bryan \& Norman 1998; Eke et al. 1998). For the parameters we employed, approximately $r_{200} \simeq 1.3 r_{\text {vir. }}$
} 
Mach numbers of $M_{s} \lesssim 2-3$ (e.g., Vazza et al. 2011), so their contribution to the production of CRs would not be significant (see the discussion in Section 2.3). Since this paper focuses on relatively strong, CR-producing shocks in the outskirts, having an uniform resolution throughout the entire simulation volume should be actually an advantage.

\subsection{Shock Identification}

A number of algorithms that can be applied to the identification of shocks in structure formation simulation data have been suggested (e.g., Ryu et al. 2003; Pfrommer et al. 2006; Skillman et al. 2008; Vazza et al. 2009a). They all employed the Rankine-Hugoniot jump conditions but in slightly different ways. Although there are some differences, the properties of identified shocks in the LSS by different algorithms are overall consistent with each other (see, e.g., Vazza et al. 2011 for a comparison study). Here we adopted the algorithm suggested by Ryu et al. (2003).

A series of the following one-dimensional procedures are first gone through for three primary directions. The grid zones are tagged as "shocked" if they fulfill all of the following conditions: (1) $\boldsymbol{\nabla} \cdot \boldsymbol{v}<0$, i.e., the local flow is converging, (2) $|\Delta \log T|>0.11$, i.e., the Mach number is greater than 1.3, and (3) $\Delta T \times \Delta \rho>0$, i.e., the gradients of temperature and density have the same sign. The central difference is defined as $\Delta Q_{i} \equiv Q_{i+1}-Q_{i-1}$ for the quantity $Q_{i}$ in the zone $i$. A shock in simulation usually spreads over several grid zones, and the "shock center" is defined as the grid zone with minimum $\boldsymbol{\nabla} \cdot \boldsymbol{v}$. While Ryu et al. (2003) used the condition $\Delta T \times \Delta s>0$ (where $s$ is the entropy), here we used the condition $\Delta T \times \Delta \rho>0$ in order to exclude the possible misidentification of contact discontinuities. We found that the current method with the condition $\Delta T \times \Delta \rho>0$ may miss some of weak shocks, but the overall statistics of identified shocks are not significantly affected. The Mach number at the shock center, $M_{s}$, is calculated by solving the relation for the gas temperature jump along the three primary directions: $T_{2} / T_{1}=\left(5 M_{s}^{2}-1\right)\left(M_{s}^{2}+3\right) /\left(16 M_{s}^{2}\right)$. Hereafter, the subscripts 1 and 2 indicate the preshock and postshock quantities, respectively. Then the Mach number of the shock center is assigned as the maximum value of the three Mach numbers, i.e., $M_{s}=\max \left(M_{s, x}, M_{s, y}, M_{s, z}\right)$. Because of complex flow patterns and shock surface topologies, very weak shocks are difficult to be identified unequivocally, so only shocks with $M_{s} \geqslant 1.5$ are considered. Hereafter, we refer to a grid zone with assigned $M_{s}$ as "a shock," which represent a small patch with an area of $(\Delta l)^{2}$. A shock surface normally consists of many of these shocks (or shock zones), so the total number of identified shocks multiplied by $(\Delta l)^{2}$ is effectively equal to the total area of shock surfaces contained in a given volume.

Once the Mach number is determined, the shock speed and the shock kinetic flux are estimated as $v_{1}=M_{s}\left(\gamma P_{\mathrm{th}, 1} / \rho_{1}\right)^{1 / 2}$ and $f_{\text {kin }}=(1 / 2) \rho_{1} v_{1}^{3}$, where $\gamma=5 / 3$ is the gas adiabatic index.

\subsection{Energy Dissipation at Shocks}

If no CRs are produced at a shock, the gas thermalization efficiency can be calculated directly from the Rankine-Hugoniot relation as $\delta_{0}\left(M_{s}\right)=\left[e_{\mathrm{th}, 2}-e_{\mathrm{th}, 1}\left(\rho_{2} / \rho_{1}\right)^{\gamma}\right] v_{2} / f_{\mathrm{kin}}$, where $e_{\mathrm{th}}$ is the internal energy density. Note that the second term inside the brackets subtracts the effect of adiabatic compression that occurs at a shock as well as the contribution of the thermal energy flux entering the shock. Then the generation of heat can be estimated with the thermal energy flux, $f_{\text {th }}=\delta_{0}\left(M_{s}\right) \times f_{\text {kin }}$. However, if

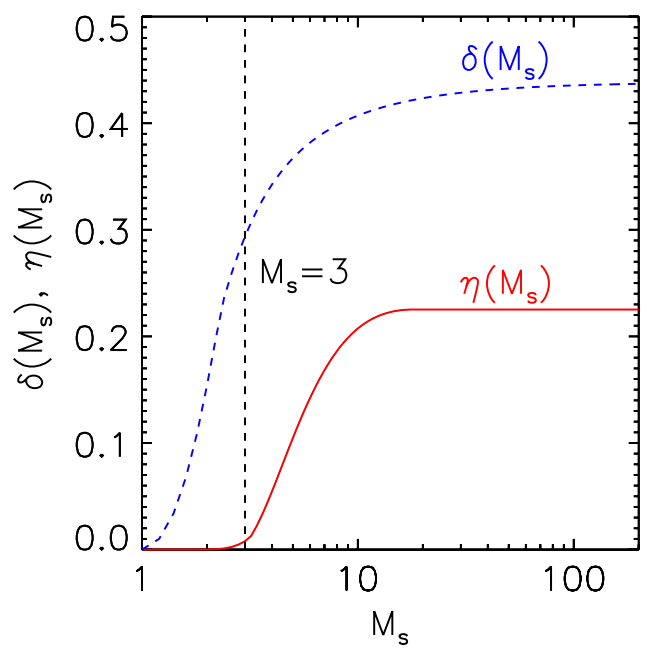

Figure 2. Gas thermalization efficiency, $\delta$ (blue dashed curve), and CRacceleration efficiency, $\eta$ (red solid curve), employed in this paper, as a function of Mach number. They were estimated with simulations of nonlinear DSA, where the upstream $\beta \equiv P_{\text {th }} / P_{B}=100$ was assumed and phenomenological models for MFA and Alfvénic drift in the shock precursor were implemented (Kang \& Ryu 2013). The vertical dashed line marks $M_{s}=3$.

(A color version of this figure is available in the online journal.)

CRs are accelerated via DSA, a fraction of the shock kinetic energy is transferred to the CR component and the resulting thermalization efficiency is reduced, i.e., $\delta\left(M_{s}\right)<\delta_{0}\left(M_{s}\right)$. With $\eta\left(M_{s}\right)$ defined as the CR-acceleration efficiency (e.g., Ryu et al. 2003; Kang \& Jones 2007), the acceleration of CR protons at shocks can be quantified with the CR energy flux, $f_{\mathrm{CR}}=\eta\left(M_{s}\right) \times f_{\text {kin }}$.

At the moment it is not possible to predict $\delta\left(M_{s}\right)$ and $\eta\left(M_{s}\right)$ from first principles, because complex wave-particle plasma interactions governing the CR injection and acceleration at collisonless shocks are not fully understood. It has been recognized that the magnetic field amplification (MFA) due to CR streaming instabilities and the Alfvénic drift of scattering centers in the amplified field play significant roles in DSA at astrophysical shocks such as supernova remnant shocks (e.g., Lucek \& Bell 2000; Bell 2004; Schure et al. 2012; Caprioli 2012; Kang 2012). Through numerical simulations of nonlinear DSA for shocks expected in the LSS, Kang \& Ryu (2013) has shown that if self-amplification of magnetic fields and fast Alfvénic drift in the shock precursor are implemented into the standard DSA theory, the CR energy spectrum is steepened and the CRacceleration efficiency is reduced, compared to the cases without including those processes. Here, we adopted $\delta\left(M_{s}\right)$ and $\eta\left(M_{s}\right)$ of Kang \& Ryu (2013).

Figure 2 shows the curves that fit the values of $\delta\left(M_{s}\right)$ and $\eta\left(M_{S}\right)$ for shocks that form in a weakly magnetized plasma with $\beta=100$ and $n_{H, 0}=10^{-4} \mathrm{~cm}^{-3}$. The high value of $\beta \sim 100$ is expected for plasmas in the ICM, as noted in Introduction. Both $\delta\left(M_{s}\right)$ and $\eta\left(M_{s}\right)$ increase as $M_{s}$ increases, and asymptote to 0.45 and 0.22 , respectively, for strong shocks with $M_{s} \gtrsim 10$. Compared to the previous estimate of $\eta \approx 0.55$ at strong shocks, given in Kang et al. (2007) where MFA and Alfvénic drift were not considered, the newly estimated CR-acceleration efficiency is smaller by a factor of $\sim 2.5$ for $M_{s} \gtrsim 10$. Also the CR acceleration is inefficient at weak shocks with $M_{s} \lesssim 3$ in our new estimation.

The steepening of CR spectrum due to Alfvénic drift and the ensuing reduction of $\eta\left(M_{s}\right)$ become important, only if the 

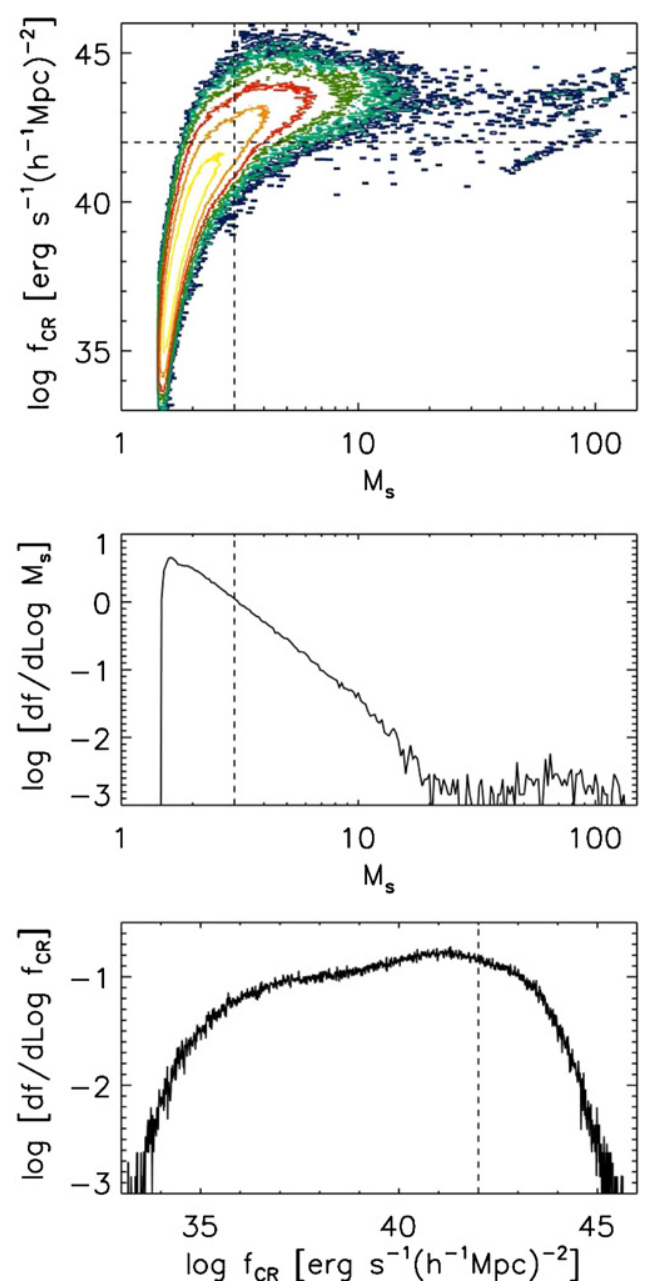

Figure 3. Top: distribution of shocks (shock zones with assigned $M_{S}$ ) found at $r \leqslant r_{200}$ in the plane of Mach number and CR energy flux. The interval of contours is a factor of $\sqrt{10}$. Middle: fraction of shocks as a function of Mach number. Bottom: fraction of shocks as a function of CR energy flux. Dashed lines mark $M_{s}=3$ and $f_{\mathrm{CR}}=10^{42} \mathrm{erg} \mathrm{s}^{-1}\left(h^{-1} \mathrm{Mpc}\right)^{-2}$. The statistics are shown for shocks identified in 134 sample clusters from $100 h^{-1} \mathrm{Mpc}$ box simulations with $1024^{3}$ and $2048^{3}$ grid zones.

(A color version of this figure is available in the online journal.)

magnetic field is strong enough so that the Alfvénic speed is substantial, that is, $V_{A} \gtrsim 0.1 v_{1}\left(M_{A} \lesssim 10\right)$, where $V_{A}$ is the Alfvénic speed in the amplified magnetic field in the shock precursor (Caprioli 2012; Kang 2012). At weak shocks $\left(M_{s} \lesssim 3\right)$, however, MFA would be inefficient, so $V_{A} \approx V_{A, 0}=$ $\sqrt{2 /(\beta \gamma)} c_{S}$, where $V_{A, 0}$ is the Alfvénic speed in the background magnetic field. For $\beta=100$, then $M_{A} \approx M_{A, 0}=v_{1} / V_{A, 0} \approx$ $10 M_{s}$, and so the Alfvénic drift effect would be only mildly important at weak shocks. At strong shocks, on the other hand, the diffusive CR pressure induces a precursor, in which the upstream flow is decelerated and adiabatically compressed, and the streaming CRs amplify significantly the turbulent magnetic fields (Bell 2004). According to the MFA prescription adopted in Kang \& Ryu (2013), the MFA factor increases with $M_{A, 0}$ and can be approximated as $B_{1} / B_{0} \approx 0.1 M_{A, 0}$, where $B_{0}$ and $B_{1}$ are the magnetic field strengths in the background medium and immediately upstream of the shock, respectively. Then, the Alfvénic Mach number defined by the amplified magnetic field becomes $M_{A, 1}=M_{A, 0}\left(B_{0} / B_{1}\right) \approx 10$ (independent of the plasma $\beta$ ), so the Alfvénic drift is expected to be important at strong CR modified shocks. We note, however, that relevant plasma physical processes, such as the injection of CRs as well as MFA and Alfvénic drift, are not well understood, so any attempts to predict the DSA efficiency involve large uncertainties, especially for weak shocks. So the dissipation efficiencies given in Figure 2 should be taken as rough estimates.

In this paper, we do not consider the re-acceleration of preexisting CRs. We note, however, that it could be important especially for weak shocks with $M_{s} \lesssim 3$ (Kang \& Ryu 2011, 2013). Kang et al. (2012) and Pinzke et al. (2013), for instance, argued that the re-acceleration of pre-existing CR electrons would be operating at radio relics associated with weak structure formation shocks.

\section{PROPERTIES AND NATURE OF SHOCKS IN CLUSTER OUTSKIRTS}

We first examine the Mach number and energetics of shocks within and around the virial radius, specifically in $r \leqslant r_{200} \approx$ $1.3 r_{\text {vir }}$, of simulated clusters. Figure 3 shows the frequency distribution of shocks (i.e., zones with assigned $M_{s}$ ) as a function of Mach number and CR energy flux for the shocks found in 134 clusters from $100 h^{-1} \mathrm{Mpc}$ box simulations with $1024^{3}$ and $2048^{3}$ grid zones. The energetics of shocks is quantified with the CR energy flux, $f_{\mathrm{CR}}$. Most of these shocks are internal shocks which are produced in the hot ICM of clusters or in the WHIM of filaments, according to the classification of Ryu et al. (2003). As previously shown, they are mostly weak with $M_{s} \lesssim 3$. The fractions of shocks with relatively high Mach numbers of $M_{s} \geqslant 3, \geqslant 4$, and $\geqslant 5$ are $\sim 19 \%, \sim 8 \%$, and $\sim 4.5 \%$, respectively, among all the shocks with $M_{s} \geqslant 1.5$ in 100 and $200 h^{-1} \mathrm{Mpc}$ box simulations. We categorize shocks or shock zones with $f_{\mathrm{CR}} \geqslant$ $10^{42} \mathrm{erg} \mathrm{s}^{-1}\left(h^{-1} \mathrm{Mpc}\right)^{-2}$ as energetic shocks. We note that the shocks responsible for observed radio relics are estimated to have the total kinetic energy flux of $\sim 10^{44}-10^{45} \mathrm{erg} \mathrm{s}^{-1}$ over the entire shock surface of $\sim\left(h^{-1} \mathrm{Mpc}\right)^{2}$ or so (e.g., van Weeren et al. 2010; Kang et al. 2012). Considering $\eta \approx 10^{-2}$ at $M_{s} \sim 3$, shocks with $f_{\mathrm{CR}} \gtrsim 10^{42} \mathrm{erg} \mathrm{s}^{-1}\left(h^{-1} \mathrm{Mpc}\right)^{-2}$ can be considered to be energetic enough to be parts of observable radio relics. The fraction of energetic shocks is $\sim 21 \%$ among all the shocks. And the fraction of shocks with $M_{s} \geqslant 3$ and $f_{\mathrm{CR}} \geqslant 10^{42} \mathrm{erg} \mathrm{s}^{-1}\left(h^{-1} \mathrm{Mpc}\right)^{-2}$ is $\sim 16 \%$.

Shocks with lower $M_{s}$ form on average close to the core with higher gas density (see Figure 4 below) and so have larger $f_{\text {kin }}$, but they have lower $\eta$. Stronger shocks, on the other hand, form mostly in the outskirts and have lower $f_{\text {kin }}$, but they have higher $\eta$. Such tendencies are reflected in the relation between $f_{\mathrm{CR}}=\eta \cdot f_{\text {kin }}$ and $M_{s}$ in Figure 3 . For weak shocks with $M_{s} \lesssim 3$, the CR-acceleration efficiency increases steeply with $M_{s}$, while the shock kinetic energy flux varies only mildly. So $f_{\text {CR }}$ increases strongly with $M_{s}$, resulting in a relatively robust correlation between the two quantities. For shocks with $M_{s} \gtrsim 3$, the dependence of $\eta$ on $M_{s}$ becomes much softer, while the variance of $f_{\text {kin }}$ increases. So the correlation between $f_{\mathrm{CR}}$ and $M_{s}$ substantially weakens. We find that shocks with the largest $f_{\mathrm{CR}}$ have typically $M_{s} \approx 3-5$, which interestingly coincides with the Mach numbers of strong radio relic shocks (see Introduction).

Figure 4 shows two-dimensional slices of three sample clusters with the X-ray emission-weighted temperatures of $T_{\mathrm{X}}=2.7 \mathrm{keV}$ (left), $2.5 \mathrm{keV}$ (middle), and $2.4 \mathrm{keV}$ (right), respectively, at present $(z=0)$. The slices were chosen to highlight the shock structures, so they pass through short comoving distances of $0.24-0.28 h^{-1} \mathrm{Mpc}$ from the cluster centers. The CR luminosity, shown in the bottom panels, is $F_{\mathrm{CR}}=f_{\mathrm{CR}}(\Delta l)^{2}$ at the comoving surfaces of shocks. Hereafter 


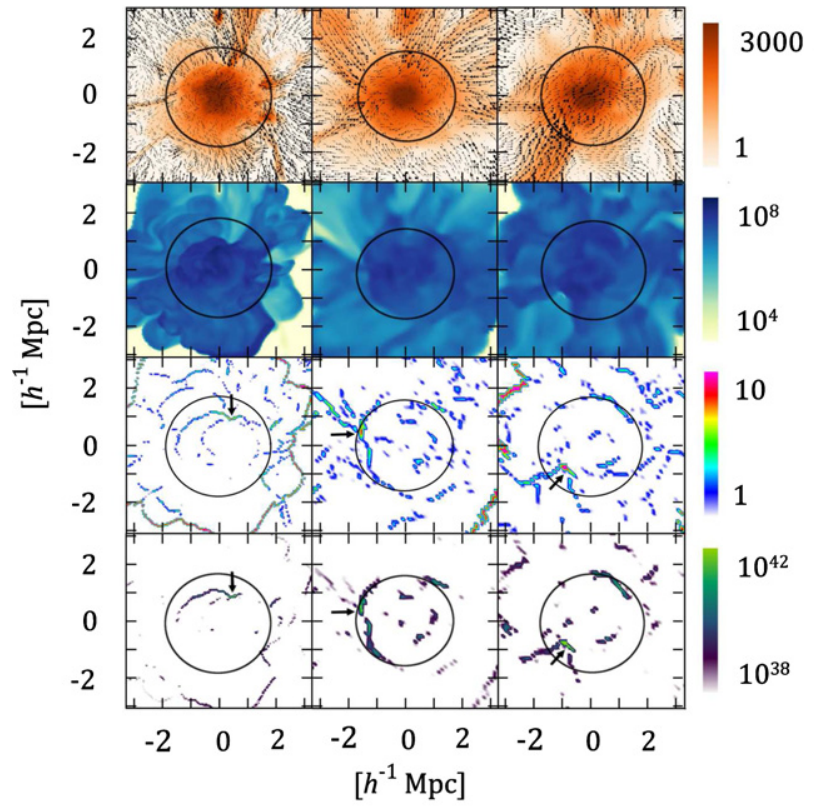

Figure 4. Two-dimensional slice images of three sample clusters showing the gas density with flow velocity field, gas temperature, shock Mach number, and CR luminosity generated at shocks (from top to bottom) at present $(z=0)$. The X-ray emission-weighted temperature of clusters is $k T_{\mathrm{X}}=2.7 \mathrm{keV}$ (left), $2.5 \mathrm{keV}$ (middle), and $2.4 \mathrm{keV}$ (right), respectively. The cluster in the left panels is from $100 h^{-1} \mathrm{Mpc}$ box simulation with $2048^{3}$ grid zones, while other two are from $100 h^{-1} \mathrm{Mpc}$ box simulations with $1024^{3}$ zones. Circles with $r=r_{200}$ are overlaid in the lower two rows. Thick arrows in the lower two rows of panels point the most energetic shocks (MESs). The MESs in the clusters shown here are infall shocks that form in the WHIM infalling along filaments.

(A color version of this figure is available in the online journal.)

the shock with the largest $f_{\mathrm{CR}}$ among shocks in each cluster will be called the most energetic shock (MES). Thick arrows in the $M_{s}$ and $F_{\mathrm{CR}}$ panels of Figure 4 point the MESs of each cluster. The MESs are located at $r \approx 1.1 h^{-1} \mathrm{Mpc}$ (left), $1.5 h^{-1} \mathrm{Mpc}$ (middle), and $1.1 h^{-1} \mathrm{Mpc}$ (right) from the cluster centers, which correspond to $0.60 r_{200}$ (left), $0.94 r_{200}$ (middle), and $0.63 r_{200}$ (right), respectively. The spatial distribution of $M_{s}$ tells that strong shocks are found in the outer regions of clusters. Obviously, the strongest shocks are external shocks that form in the accreting gas from voids $\left(T \approx 10^{4} \mathrm{~K}\right.$; see Ryu et al. 2003). However, as noted in the Introduction, owing to low density, they are energetically unimportant, so we are not concerned about those external shock in this paper. Energetic shocks that produce large amounts of CRs are internal shocks and they reside mostly in the outskirts of the clusters, as shown in the distribution of $F_{\mathrm{CR}}$.

The distributions of $\rho, v, T$, and $M_{s}$ indicate that the structures including the MESs in Figure 4 look like infall shocks that form by the infall of gas from filaments. Those infall shocks are energetic enough to penetrate into the region inside the virial radius where the gas density is relatively high, indicating that their shock kinetic energy flux is large. They are also relatively strong with $M_{s} \sim 5-7$, so they are efficient CR accelerators. These characteristics make the infall shocks the MESs in the clusters shown here. We point that not all filaments induce infall shocks inside the virialized regions of clusters. Also the cross sectional areas of penetrated filaments are small, compared to the surface area of virialized regions $\sim 4 \pi r_{\mathrm{vir}}^{2}$. So the energetic infall shocks inside the virial radius should account for a small fraction of internal shocks in the ICM. The distribution of $F_{\mathrm{CR}}$

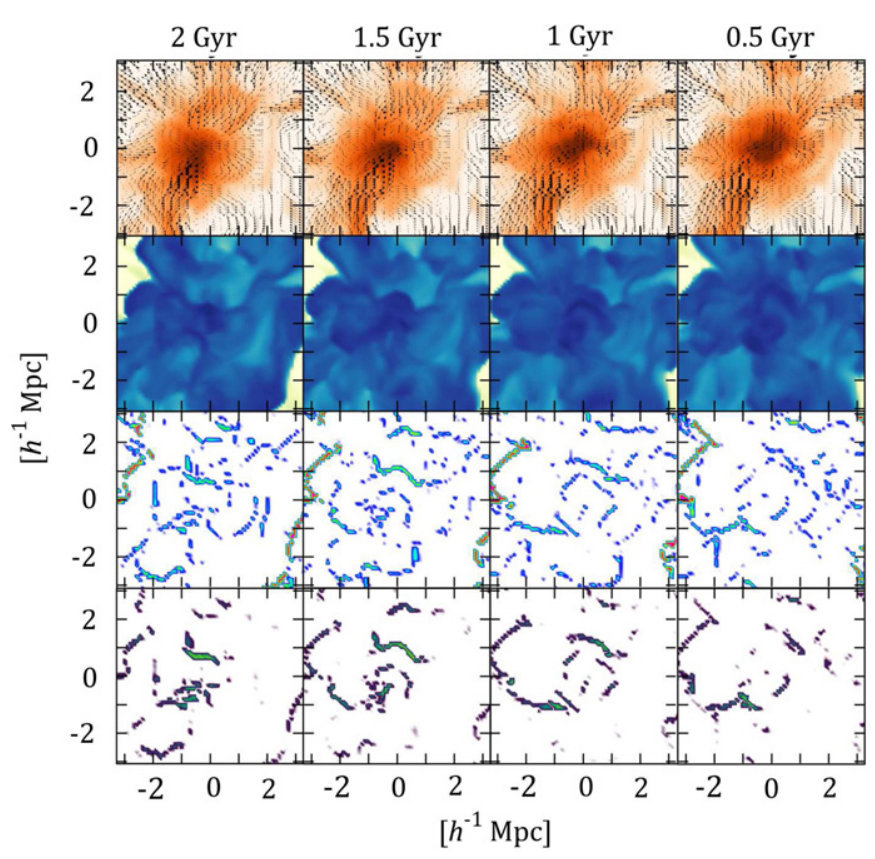

Figure 5. Time evolution of the cluster shown in the right column of Figure 4. The numbers at the top are the look-back times. Shown are also the gas density with flow velocity field, gas temperature, shock Mach number, and CR luminosity generated at shocks (from top to bottom). The color bars (not shown) are the same as those in Figure 4.

(A color version of this figure is available in the online journal.)

in Figure 4, however, indicates that infall shocks in the outskirts could be responsible for a significant fraction of $\mathrm{CR}$ production in the clusters (see below).

Figure 5 shows the time evolution of the cluster shown in the right column of Figure 4, demonstrating how the cluster has evolved dynamically and how various types of shocks have been induced. Strong external shocks persist at the comoving distance 3-5 $h^{-1} \mathrm{Mpc}$ away from the cluster center, while numerous internal shocks form and disappear in a dynamical timescale of $\sim 1$ Gyr. One can see that the cluster experienced a merger at the look-back time of $2 \times 10^{9} \mathrm{yr}$, producing several merger shocks. Then infall from an attached filament in the southwest direction followed, and penetrated into the core region. It was halted by an energetic infall shock that developed around the look-back time of $1 \mathrm{Gyr}$ and lasted to the present epoch.

To quantify the statistics of infall shocks, we separated them from other shocks. Infall shocks are defined as those that decelerate the WHIM accreting from filaments to a cluster (see the Introduction). So we employed the following criteria for infall shocks in $r \leqslant r_{200}$ using the entropy and density of the preshock gas and the sonic Mach number: (1) $\log \left[T_{1}(\mathrm{~K}) /\left(\rho_{1} /\langle\rho\rangle\right)^{2 / 3}\right] \leqslant$ 5.3, (2) $\rho_{1} /\langle\rho\rangle \leqslant 10^{3}$, and (3) $M_{s} \geqslant 3$. Note that the first criterion is an entropy condition. Figure 6 shows the volumetric distribution of the gas from $100 h^{-1} \mathrm{Mpc}$ box simulations with $1024^{3}$ grid zones. The domain demarcated by the first and second criteria does not coincide with the conventional definition of the WHIM, $10^{5} \mathrm{~K} \leqslant T \leqslant 10^{7} \mathrm{~K}$. However, visual inspections indicated that the above three criteria pick up infall shocks in the region of $r \leqslant r_{200}$ best among different criteria we have tried. Figure 7 shows a slice displaying the positions of shocks identified as infall shocks according to the above criteria as well as those which are not infall shocks, for the cluster shown in the right column of Figure 4. We note that the morphology of shock surfaces could be quite complicated, depending on the 


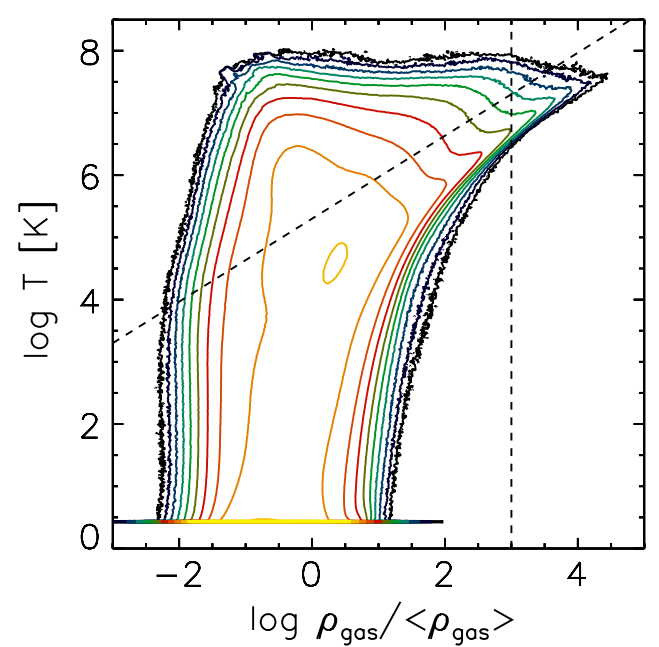

Figure 6. Volumetric distribution of the gas in the plane of density and temperature from $100 h^{-1} \mathrm{Mpc}$ box simulations with $1024^{3}$ grid zones. The diagonal and vertical dashed lines mark $\log \left[T(K) /(\rho /\langle\rho\rangle)^{2 / 3}\right]=5.3$ and $\rho /\langle\rho\rangle=10^{3}$, respectively. In our classification scheme, infall shocks have the preshock gas that is characterized by the following conditions: $\log \left[T(K) /(\rho /\langle\rho\rangle)^{2 / 3}\right] \leqslant 5.3$ and $\rho /\langle\rho\rangle \leqslant 10^{3}$ (see the main text for details).

(A color version of this figure is available in the online journal.)

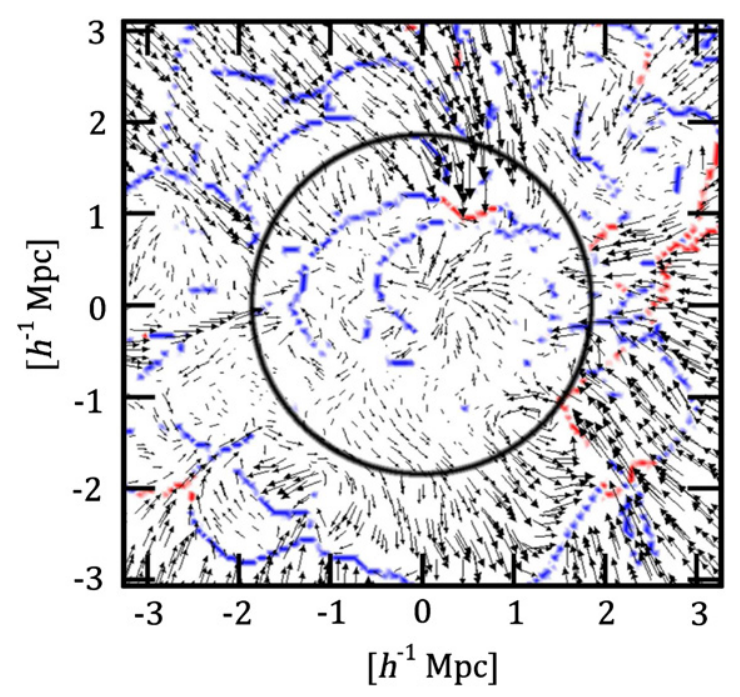

Figure 7. Slice displaying the positions of infall shocks (red) and not-infall shocks (blue) according to the criteria in Section 3, along with the flow velocity field, for the cluster shown in the right column of Figure 4. The circle marks $r=r_{200}$.

(A color version of this figure is available in the online journal.)

dynamical history of clusters. In the cluster shown, for instance, the infall shock including the MES is connected to a larger shock surface, a portion of which is a (not-infall) shock expanding from the core to the outskirt. In general a connected shock surface can consist of a number of infall and not-infall shocks.

With the above criteria, we found that, among all the shocks with $M_{s} \geqslant 1.5$ located in $r \leqslant r_{200}$ of the sample clusters, about $10 \%$ are classified as infall shocks. So most of ICM shocks are merger shocks or turbulent shocks (see Introduction). As noted in Figure 3, 19\% of the identified shocks have $M_{s} \geqslant 3$, so about a half $(\sim 55 \%)$ of them are infall shocks. Identifying merger shocks would require the examination of the time evolution of cluster dynamics, which we did not attempt here.

Among the energetic shocks with $f_{\mathrm{CR}} \geqslant 10^{42} \mathrm{erg} \mathrm{s}^{-1}$ $\left(h^{-1} \mathrm{Mpc}\right)^{-2}$, the fraction of infall shocks is $\sim 44 \%$. Among
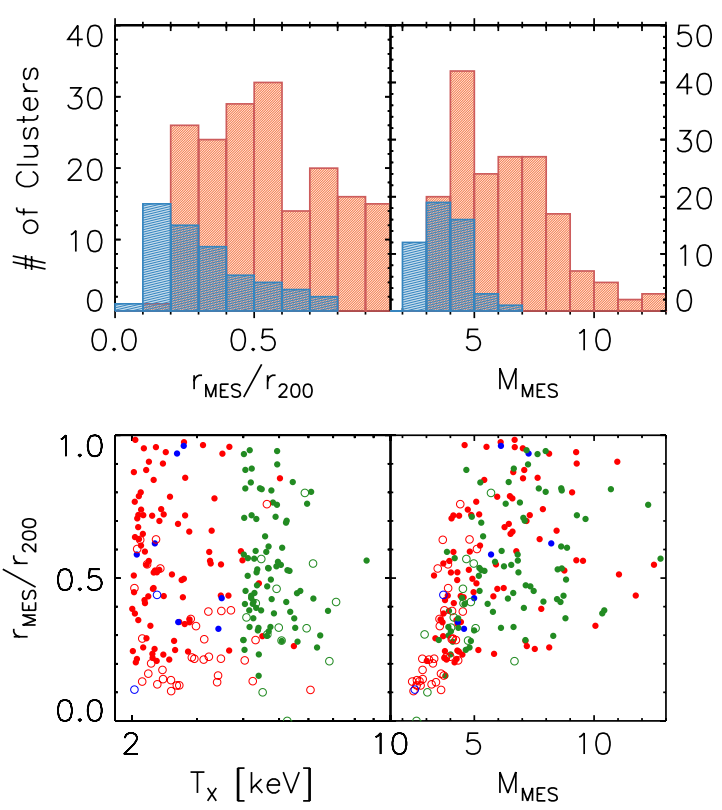

Figure 8. Top: frequency distributions of radial positions, $r_{\mathrm{MES}}$ (left), and Mach numbers, $M_{\text {MES }}$ (right), of the most energetic shocks (MESs) in our 228 sample clusters. Red histogram is for 177 clusters where the MESs are infall shocks, and blue histogram is for 51 clusters where the MESs are not infall shocks. Bottom: $r_{\mathrm{MES}}$ as a function of cluster temperature (left) and $M_{\mathrm{MES}}$ (right). Red dots denote the clusters from $100 h^{-1}$ Mpc box simulations with $1024^{3}$ grid zones, green dots denote the clusters from $200 h^{-1} \mathrm{Mpc}$ box simulations with $1024^{3}$ zones, and blue dots denote the clusters from $100 \mathrm{~h}^{-1} \mathrm{Mpc}$ box simulation with $2048^{3}$ zones, respectively. Filled dots are for 177 clusters in which the MESs are infall shocks, while open dots are for 51 clusters in which the MESs are not infall shocks.

(A color version of this figure is available in the online journal.)

the MESs in 228 sample clusters, 177 are infall shocks; i.e., $\sim 78 \%$ of the MESs are infall shocks in our sample. We expect the MESs that are not infall shocks to be merger shocks. The top panels of Figure 8 show the distributions of the radial position, $r_{\mathrm{MES}}$, and the Mach number, $M_{\mathrm{MES}}$, of the MESs. The MESs that are infall shocks (red histogram, MES-ISs hereafter) are distributed over all radius, peaking at $r_{\mathrm{MES}} / r_{200} \sim 0.5$. On the other hand, the MESs that are not infall shocks (blue histogram, MES-NISs hereafter) are mostly found at inner parts of clusters. For the MES-ISs, the Mach number distribution peaks around $4 \lesssim M_{\mathrm{MES}} \lesssim 5$, and decreases sharply for smaller $M_{\mathrm{MES}}$ but extends to larger $M_{\mathrm{MES}}$. For the MES-NISs, the Mach number distribution is mostly limited to $M_{\mathrm{MES}} \lesssim 5$. The figure demonstrates that the MES-ISs are found mainly at outer parts of clusters and they have higher Mach numbers than the MESNISs, as expected. We attempt to find correlations among $r_{\mathrm{MES}}$, $T_{\mathrm{X}}$, and $M_{\mathrm{MES}}$ in the bottom panels of Figure 8. It appears that there is no noticeable correlation between $r_{\mathrm{MES}}$ and $T_{\mathrm{X}}$. But $r_{\mathrm{MES}}$ tends to be larger at larger $M_{\mathrm{MES}}$, confirming that higher Mach number shocks form at outer regions of clusters.

\section{COSMIC RAY PRODUCTION AT SHOCKS IN CLUSTER OUTSKIRTS}

We next examine the spatial characteristics of the CR proton production in clusters. Figure 9 shows the radial distributions of the maximum Mach number, $M_{s, \max }$, the CR luminosity per unit radius, $L_{\mathrm{CR}}$, and the fraction of CR luminosity due to infall shocks, $L_{\mathrm{CR} \text {,infall }} / L_{\mathrm{CR}}$, in four sample clusters. Here, $M_{s, \max }$ is defined as the highest Mach number of shocks located in the bin of $[r, r+d r]$, while $L_{\mathrm{CR}}$ is the sum of $F_{\mathrm{CR}}$ of shocks in the bin 


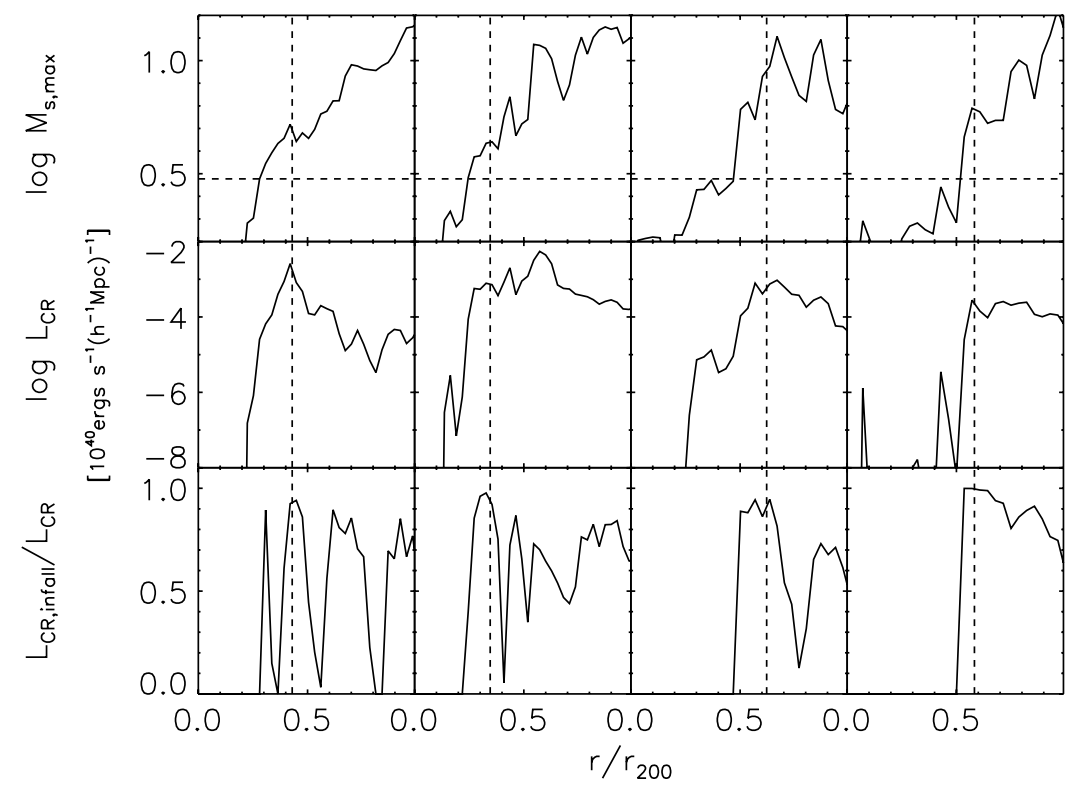

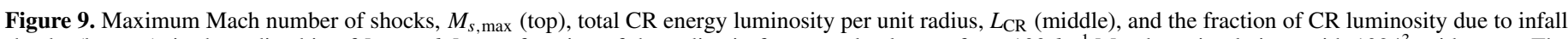

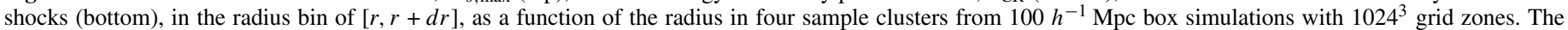

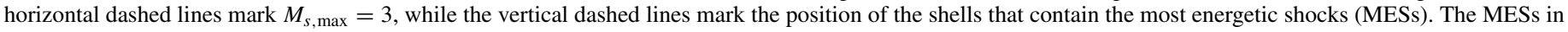
the clusters shown here are all infall shocks.

divided by the width $d r$. Note that $L_{\mathrm{CR}}$ represents the amount of $\mathrm{CR}$ energy produced per unit time per unit length and has a rather unusual unit, erg s $\mathrm{s}^{-1}\left(h^{-1} \mathrm{Mpc}\right)^{-1} .{ }^{9}$ The vertical dashed lines mark the radial bins that contain the MESs.

The distribution of $M_{s, \max }$ demonstrates that on average shocks tend to be stronger in the outer regions of clusters, as expected. The CR luminosity $L_{\mathrm{CR}}$ is dominated by energetic shocks in a given radial bin, and energetic shocks are found mostly in the outskirts, so $L_{\mathrm{CR}}$ is higher there. The MESs and the peaks of $L_{\mathrm{CR}}$ are located in $r \gtrsim 0.4 r_{200}$ (although the two do not necessarily coincide), indicating that CRs are produced mostly at the outer regions of clusters. These findings are consistent with the previous studies using the structure formation simulations in which the production of CR protons was explicitly followed in runtime (Pfrommer et al. 2007; Vazza et al. 2012). With high $\mathrm{CR}$ production at the outskirts, we expect the radial profile of the CR pressure is flatter than that of the gas pressure and could be even inverted (see Brunetti et al. 2012), as mentioned in the Introduction.

The distribution of $L_{\mathrm{CR} \text {,infall }} / L_{\mathrm{CR}}$ shows that infall shocks contribute to the $\mathrm{CR}$ production by a large fraction, especially in the outskirts. As a matter of fact, we estimate that infall shocks produce $\sim 68 \%$ of CRs in $r \leqslant r_{200}$, when summed for all 228 clusters in our sample. Recall that the fraction of infall shocks among those with $M_{s} \geqslant 3$ inside the volume of $r \leqslant r_{200}$ is $\sim 55 \%$.

We also examine the momentum distribution of the CR protons, which are expected to be produced in the outskirts and then mixed in the ICM via turbulent flow motions (see Introduction). For each shock with $M_{s}$, we adopted the testparticle power-law distribution function, $f_{p}(p) \propto p^{-q}$, where $q=4 M_{s}^{2} /\left(M_{s}^{2}-1\right)$ (Drury 1983). Note that the CR acceleration at cluster shocks with $M_{s} \lesssim 5$ is reasonably described by

\footnotetext{
9 The volume-averaged CR energy production rate per unit volume at given radius is $L_{\mathrm{CR}} / 4 \pi r^{2}$. Due to the large range of $L_{\mathrm{CR}}$, the radial distribution of $L_{\mathrm{CR}} / 4 \pi r^{2}$ looks similar to that of $L_{\mathrm{CR}}$.
}

the test-particle solution (Kang \& Ryu 2010). In our sample, $\sim 96 \%$ of shocks have $M_{s} \leqslant 5$ and shocks with the largest $f_{\text {CR }}$ have typically $M_{s} \approx 3-5$ (see Section 3 ). So the testparticle solution should give reasonable results. For each sample cluster, the volume-integrated, momentum distribution function of $\mathrm{CR}$ protons within the radius $r, f_{p}(p,<r)$, is estimated as follows: (1) at each shock zone $i$, the power-law function, $f_{p, i}(p)=f_{0} p^{-q}$, is normalized with the CR energy flux at the shock as $f_{\mathrm{CR}, i}=4 \pi m_{p} c^{2} \cdot v_{2} \int_{p_{\min }}^{p_{\max }}\left(\sqrt{p^{2}+1}-1\right) f_{p, i}(p) d^{3} p$. Here, $p$ is expressed in unit of $m_{p} c$, and $p_{\min }=10^{-2} m_{p} c$ and $p_{\max }=\infty$ are assumed. (2) The volume-integrated momentum distribution function is calculated by adding up $f_{p, i}$ for all shocks inside $r$, i.e., $f_{p}(p,<r)=\sum_{r_{i}<r} f_{p, i}(p)$. (3) Then, the slope of the integrated momentum distribution function is estimated by fitting $f(p,<r)$ to a power-law form with the slope $\bar{q}(<r)$.

The left panel of Figure 10 shows the values of $\bar{q}(<r)$ calculated for some of sample clusters. ${ }^{10}$ The average value of $\bar{q}(<r)$ decreases with $r$, reflecting the fact that shocks are stronger on average in the outer regions of clusters, and the variation of its distribution also decreases with $r$. The average values, $\langle\bar{q}(<r)\rangle \approx 5.5$ at $r=0$ and $\langle\bar{q}(<r)\rangle \approx 4.35$ at $r=r_{\mathrm{vir}}$, correspond to the DSA power-law slopes for shocks with $M_{s} \approx 2$ and 3.5, respectively. At $r=r_{200}$, the values spread over a narrow range of $\bar{q}\left(<r_{200}\right) \approx 4.25-4.5$, corresponding to $M_{s} \approx 3-4$, as shown in the right panel of Figure 10. The plot indicates that $\bar{q}\left(<r_{200}\right)$ does not have any noticeable correlation with the cluster temperature (nor with the cluster mass and radius although not shown). These imply that the averaged Mach number of shocks, weighted with $C R$ production, in our sample clusters is in the range of $\left\langle M_{s}\right\rangle_{\mathrm{CR}} \approx 3-4$, regardless of the properties of clusters.

\footnotetext{
10 The profile of $\bar{q}(r)$, the slope of the momentum distribution of CR protons produced at shocks in the bin of $[r, r+d r]$, is similar to that of $\bar{q}(<r)$, since the $\mathrm{CR}$ production is dominated by shocks in the outer regions of clusters.
} 


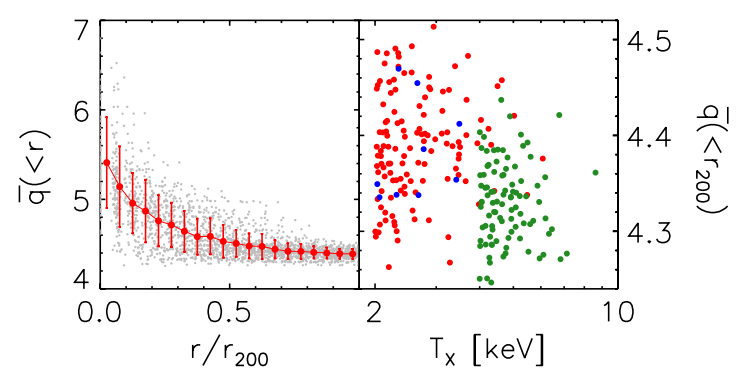

Figure 10. Left: power-law slope of the momentum distribution of the CR protons produced at shocks inside the sphere of radius $r$ as a function of $r$. Gray dots are the data calculated at different radii in some of our sample clusters. Red filled circles and error bars show their averages and $1 \sigma$ deviations in given radius bins, respectively. Right: power-law slope of the momentum distribution of the CR protons produced at shocks inside the sphere of $r_{200}$ as a function of cluster temperature. Red dots denote the clusters from $100 h^{-1} \mathrm{Mpc}$ box simulations with $1024^{3}$ grid zones, green dots denote the clusters from $200 \mathrm{~h}^{-1} \mathrm{Mpc}$ box simulations with $1024^{3}$ zones, and blue dots denote the clusters from $100 h^{-1} \mathrm{Mpc}$ box simulation with $2048^{3}$ zones, respectively.

(A color version of this figure is available in the online journal.)

\section{DISCUSSION}

The existence of $\mathrm{CR}$ protons in galaxy clusters remains to be confirmed directly from observations. CR protons produce $\gamma$-ray radiation through $p-p$ collisions with background thermal protons, but so far only upper limits on $\gamma$-ray fluxes from clusters have been set by Fermi-LAT and VERITAS, as noted in the Introduction (Ackermann et al. 2010, 2013; Arlen et al. 2012). On the other hand, secondary electrons are also produced through $p-p$ collisions, and they along with $\mu \mathrm{G}$-level magnetic fields emit the synchrotron radiation in radio over the cluster scale. The observed radio emission is produced typically by electrons with energy of several $\mathrm{GeV}$ corresponding to the Lorentz factor of $\gamma_{e} \sim 10^{4}$ (Kang et al. 2012). For this energy range, the secondary electrons at production have the momentum distribution similar to the proton spectrum, that is, $f_{e}^{p}(p) \propto p^{-q_{e}^{p}}$ with $q_{e}^{p} \approx q$ (e.g., Dermer 1986). For the proton power-law of $\bar{q} \approx 4.25-4.5$ estimated in the previous section, the secondary electron slope is also $q_{e}^{p} \approx 4.25-4.5$. Relativistic electrons suffers radiative coolings, dominantly by synchrotron and inverse Compton losses. The cooling timescale of electrons of $\gamma_{e} \sim 10^{4}$ is $\tau_{\text {cool }} \sim 10^{8} \mathrm{yr}$ with cluster magnetic fields of a few $\mu \mathrm{G}$ (e.g., Kang et al. 2012). The momentum distribution function of the secondary electrons at the steady state governed by the production through $p-p$ collisions and the cooling processes is given as $f_{e}^{s s}(p) \propto p^{-q_{e}^{s s}}$ with $q_{e}^{s s}=q_{e}^{p}-1$ (Dolag \& Enßlin 2000). So for $\bar{q} \approx 4.25-4.5$, the secondary electron slope becomes $q_{e}^{s s} \approx 5.25-5.5$.

Radio halos associated with some galaxy clusters are explained as diffuse synchrotron emissions over the cluster scale. The spectral index of observed radio halos is typically in the range of $\alpha_{R} \approx 1-1.5$, although in some radio halos it is much steeper (Feretti et al. 2012). For $\alpha_{R}=\left(q_{e}-3\right) / 2$, this requires the existence of relativistic electrons with the power slope $q_{e} \approx 5-6$, which nicely embraces the slope of steady-state secondary electrons described above. In the so-called hadronic model, for instance, the $\mathrm{CR}$ electrons emitting synchrotron radiation are assumed to be secondaries produced through $p-p$ collisions (e.g., Dolag \& Enßlin 2000; Pfrommer \& Enßlin 2004). Our results indicate that the $\mathrm{CR}$ protons accelerated at shocks in the cluster outskirts may be capable of producing secondary electrons with the right energy spectral slope $\left(q_{e}^{s s} \approx 5.25-5.5\right)$ for the spectral index of observed radio halos. Brunetti et al.
(2012), however, argued that at least for the Coma cluster, the hadronic model that requires the $\mathrm{CR}$ proton energy $\gtrsim 3 \%-5 \%$ of the thermal energy may violate the $\gamma$-ray upper limit set by Fermi-LAT observations, provided that the magnetic field is not much stronger than that measured/constrained by Faraday RM. Moreover, according to a more recent Fermi-LAT paper (Ackermann et al. 2013), this limit has become even more stringent, constraining the $\mathrm{CR}$ proton energy down to $\lesssim 1 \%$ of the thermal energy in clusters. In the so-called re-acceleration model, on the other hand, the secondary electrons are further accelerated by turbulence in the ICM, so the CR proton requirement is alleviated somewhat (e.g., Brunetti et al. 2001). The detailed implications of our results for radio halo are complicated, and addressing them properly requires studies which are beyond the scope of this paper.

So far, our discussions on DSA at energetic shocks have been focused mostly on CR protons and secondary electrons resulted from $p-p$ collisions. As for supernova remnant shocks, primary $\mathrm{CR}$ electrons can be accelerated at ICM shocks in the same manner as CR protons, although the injection (preacceleration) of electrons into DSA process remains rather uncertain. We point that the projected surfaces of energetic shocks would have morphologies of partial shells or elongated arcs (see Figure 4), so diffuse synchrotron emissions from primary CR electrons accelerated at these shocks could produce radio structures that resemble radio relics discovered in the cluster outskirts (e.g., van Weeren et al. 2010; Kang et al. 2012). Moreover, the average radial distance of the MESs in Figure 8 is $\left\langle r_{\text {MES }}\right\rangle \sim 0.5 r_{200} \sim 0.5-1.5 h^{-1} \mathrm{Mpc}$, which is comparable to the average distance of observed radio relics from the cluster center (e.g., van Weeren et al. 2009). So, for instance, radio relics with flat radio spectrum such as the sausage relic in CIZA J2242.8+5301 $\left(\alpha_{R} \approx 0.6\right)$ could be explained by primary electrons accelerated by energetic shocks (a substantial fraction of which are infall shocks) in the cluster outskirts.

In addition, pre-existing CR electrons in the ICM (previously produced at shocks or through $p-p$ collisions) can be reaccelerated at energetic shocks (Kang et al. 2012; Pinzke et al. 2013). To explain the observed properties of radio relics with flat radio spectra, Kang et al. (2012), for instance, proposed a model in which pre-existing CR electrons with the momentum distribution corresponding to the observed radio spectral index are re-accelerated at weak shocks with $M_{s} \lesssim 2-3$. The sausage relic in CIZA J2242.8+5301 then requires pre-existing CR electrons with $q_{e} \approx 4.2$. It is interesting to note that this is close to the slope of the secondary electrons at production $\left(q_{e}^{p} \approx 4.25-4.5\right)$ due CR protons accelerated at energetic shocks in the cluster outskirts. The electrons with $\gamma_{e} \lesssim 10^{2}$ have the cooling time longer than the age of the universe. So we may conjecture a scenario in which the secondary electrons produced with $\gamma_{e} \lesssim 10^{2}$ are boosted to $\gamma_{e} \gtrsim 10^{4}$ at shocks in the cluster outskirts, producing radio-relic-like structures. The acceleration or re-acceleration of CR electrons at shocks in clusters, compared to those of CR protons, involve additional complications such as injection, pre-existing CR population, and cooling.

\section{SUMMARY}

The outskirts of galaxy clusters are dynamically active, reflecting disturbances due to mergers of substructures and continuous infall of gas along filaments. A manifestation of such activities is the formation of shock waves, which can be observed in radio and X-ray. In this paper, we have studied 
structure formation shocks in the cluster outskirts. Specifically, in a sample of 228 clusters from numerical simulations of the LSS formation with uniform grid resolution, we have identified the ICM shocks located in $r \leqslant r_{200}\left(\approx 1.3 r_{\text {vir }}\right)$ and studied their properties and the CR proton production via DSA there.

Our main results are summarized as follows.

1. As previously known (e.g., Ryu et al. 2003), the ICM shocks existing in $r \leqslant r_{200}$ are mostly weak. However, there are a number of shocks that are strong and energetic enough to produce substantial amounts of CR protons via DSA. Shocks with $M_{s} \approx 3-5$ produce the largest amount of CR protons. Among shocks with $M_{s} \geqslant 1.5$, the fractions of shocks (actually shock zones) with $M_{s} \geqslant 3$, $\geqslant 4$, and $\geqslant 5$ are $\sim 19 \%, \sim 8 \%$, and $\sim 4.5 \%$, respectively, in our sample. Shocks with the CR energy flux $f_{\mathrm{CR}} \geqslant$ $10^{42} \mathrm{erg} \mathrm{s}^{-1}\left(h^{-1} \mathrm{Mpc}\right)^{-2}$, were categorized as energetic shocks. The fraction of energetic shocks (again shock zones) is $\sim 21 \%$ of the identified shocks. The shock with the largest $f_{\mathrm{CR}}$ in each cluster was designated as the MES.

2. Infall shocks, which form by the infall of the WHIM from filaments, were separated from other shocks by employing the entropy, density, and Mach number criteria. In $r \leqslant r_{200}$, $\sim 10 \%$ of shocks with $M_{s} \geqslant 1.5$ and about a half of shocks with $M_{s} \geqslant 3$ are classified as infall shocks. Infall shocks are not as common as merger or turbulent shocks, but with relatively high Mach numbers $\left(M_{s} \gtrsim 3\right)$ and large kinetic energy fluxes, they contribute to a significant fraction of CR production in clusters. We found that $\sim 44 \%$ of energetic shocks and $\sim 78 \%$ of the MESs are classified as infall shocks. And infall shocks produce $\sim 68 \%$ of CRs in $r \leqslant r_{200}$, when summed for all clusters in our sample.

3. Strong energetic shocks, including infall shocks, reside mostly in the cluster outskirts. Hence, CR protons are expected to be produced mostly in the outskirts and then advected into the core regions along with flow motions. The advection timescale is a substantial fraction of the age of the universe. Consequently, the radial profile of the CR pressure is expected to be broad, dropping off more slowly than that of the gas pressure, and might be even temporarily inverted peaking in the outskirts, as shown in previous simulations (e.g., Pfrommer et al. 2007; Vazza et al. 2012).

4. We have estimated the momentum distribution of the CR protons produced at shocks in $r \leqslant r_{200}$. The volumeintegrated momentum spectrum has the power-law slope of $q_{p} \approx 4.25-4.5$. So the average Mach number of shocks in our sample clusters, weighted with $\mathrm{CR}$ production, is in the range of $\left\langle M_{s}\right\rangle_{\mathrm{CR}} \approx 3-4$. It is greater than the characteristic Mach numbers of merger shocks $\left(M_{s} \approx\right.$ $2-3)$. This confirms that a substantial fraction of CRs are produced by energetic infall shocks in the cluster outskirts.

5. We suggest that some radio relics with flat radio spectrum such as the sausage relic in CIZA J2242.8+5301 $\left(\alpha_{R} \approx 0.6\right)$ could be explained by primary electrons accelerated by energetic infall shocks with $M_{s} \gtrsim 3$ induced in the cluster outskirts.

The implications of our results on radio observations of clusters were briefly discussed, leaving detailed studies for future works.

We thank the anonymous referee for constructive comments. We also thank G. Brunetti and T. W. Jones for comments on the manuscript. H.K. thanks V. Petrosian and KIPAC for their hospitality during the sabbatical leave at Stanford University, where a part of work was done. S.E.H. was supported by the National Research Foundation of Korea through grant 20070093860. D.R. was supported by research fund of Chungnam National University. H.K. was supported by Basic Science Research Program through the National Research Foundation of Korea (NRF) funded by the Ministry of Education, Science and Technology (2012-001065). R.C. was supported in part by grant NASA NNX11AI23G. Computing resources were in part provided by the NASA High-End Computing (HEC) Program through the NASA Advanced Supercomputing (NAS) Division at Ames Research Center.

\section{REFERENCES}

Ackermann, M., Ajello, M., Albert, A., et al. 2013, ApJ, submitted (arXiv:1308.5654)

Ackermann, M., Ajello, M., Allafort, A., et al. 2010, ApJL, 717, L71

Akamatsu, H., \& Kawahara, H. 2013, PASJ, 65, 16

Akamatsu, H., Takizawa, M., Nakazawa, K., et al. 2012, PASJ, 64, 67

Arlen, T., Aune, T., Beilicke, M., et al. 2012, ApJ, 757, 123

Bell, A. R. 1978, MNRAS, 182, 147

Bell, A. R. 2004, MNRAS, 353, 550

Berezinsky, V. S., Blasi, P., \& Ptuskim, V. S. 1997, ApJ, 487, 529

Blandford, R. D., \& Ostriker, J. P. 1978, ApJL, 221, L29

Bonafede, A., Giovannini, G., Feretti, L., Govoni, F., \& Murgia, M. 2009, A\&A, 494, 429

Brown, S., \& Rudnick, L. 2011, MNRAS, 412, 2

Brüggen, M., Bykov, A., Ryu, D., \& Röttgering, H. 2012, SSRv, 166, 187

Brunetti, G., Blasi, P., Reimer, O., et al. 2012, MNRAS, 426, 956

Brunetti, G., Setti, G., Feretti, L., \& Giovannini, G. 2001, MNRAS, 320, 365

Bryan, G. L., \& Norman, M. L. 1998, ApJ, 495, 80

Caprioli, D. 2012, JCAP, 07, 038

Cen, R., \& Chisari, N. E. 2011, ApJ, 731, 11

Clarke, T. E., \& Enßlin, T. A. 2006, AJ, 131, 2900

Dermer, C. D. 1986, A\&A, 157, 223

Dolag, K., \& Enßlin, T. A. 2000, A\&A, 362, 151

Drury, L. O'C. 1983, RPPh, 46, 973

Eke, V. R., Navarro, J. F., \& Frenk, C. S. 1998, ApJ, 503, 569

Enßlin, T., Pfrommer, C., Miniati, F., \& Subramanian, K. 2011, A\&A, 527, A99

Feretti, L., Giovannini, G., Govoni, F., \& Murgia, M. 2012, A\&ARv, 20, 54

Gabici, S., \& Blasi, P. 2003, ApJ, 583, 695

George, M. R., Fabian, A. C., Sanders, J. S., Young, A. J., \& Russell, H. R. 2009, MNRAS, 395, 657

Hoeft, M., Brüggen, M., Yepes, G., Gottlober, S., \& Schwope, A. 2008, MNRAS, 391,1511

Kang, H. 2012, JKAS, 45, 127

Kang, H., Cen, R., Ostriker, J. P., \& Ryu, D. 1994, ApJ, 428, 1

Kang, H., \& Jones, T. W. 2007, APh, 28, 232

Kang, H., \& Ryu, D. 2010, ApJ, 721, 886

Kang, H., \& Ryu, D. 2011, ApJ, 734, 18

Kang, H., \& Ryu, D. 2013, ApJ, 764, 95

Kang, H., Ryu, D., Cen, R., \& Ostriker, J. P. 2007, ApJ, 669, 729

Kang, H., Ryu, D., \& Jones, T. W. 2012, ApJ, 756, 97

Komatsu, E., Smith, K. M., Dunkley, J., et al. 2011, ApJS, 192, 18

Lau, E. T., Kravtsov, A. V., \& Nagai, D. 2009, ApJ, 705, 1129

Li, S., Li, H., \& Cen, R. 2008, ApJS, 174, 1

Lucek, S. G., \& Bell, A. R. 2000, MNRAS, 314, 65

Markevitch, M., Forman, W. R., Sarazin, C. L., \& Vikhlinin, A. 1998, ApJ, 503, 77

Markevitch, M., Gonzalez, A. H., David, L., et al. 2002, ApJ, 567, 27

Markevitch, M., \& Vikhlinin, A. 2007, PhR, 443, 1

Miniati, F., Ryu, D., Kang, H., \& Jones, T. W. 2001, ApJ, 559, 59

Miniati, F., Ryu, D., Kang, H., et al. 2000, ApJ, 542, 608

Nakamura, T. T., \& Suto, Y. 1997, PThPh, 97, 49

Nuza, S. E., Hoeft, M., van Weeren, R. J., Gottlöber, S., \& Yepes, G. 2012, MNRAS, 420, 2006

Ogrean, G. A., \& Brüggen, M. 2013, MNRAS, 433, 1701

Ogrean, G. A., Brüggen, M., van Weeren, R. J., et al. 2013, MNRAS, 433, 812

Peebles, P. J. E. 1980, The Large-Scale Structure of the Universe (Princeton, NJ: Princeton Univ. Press)

Pfrommer, C., \& Enßlin, T. A. 2004, MNRAS, 352, 76

Pfrommer, C., Enßlin, T. A., Springel, V., Jubelgas, M., \& Dolag, K. 2007, MNRAS, 378, 385 
Pfrommer, C., \& Jones, T. W. 2011, ApJ, 730, 22

Pfrommer, C., Springel, V., Enßlin, T. A., \& Jubelgas, M. 2006, MNRAS, 367,113

Pinzke, A., Oh, S. P., \& Pfrommer, C. 2013, MNRAS, 435, 1061

Russell, H. R., Sanders, J. S., Fabian, A. C., et al. 2010, MNRAS, 406, 1721

Ryu, D., Kang, H., Cho, J., \& Das, S. 2008, Sci, 320, 909

Ryu, D., Kang, H., Hallman, E., \& Jones, T. W. 2003, ApJ, 593, 599

Ryu, D., Ostriker, J. P., Kang, H., \& Cen, R. 1993, ApJ, 414, 1

Ryu, D., Schleicher, D. R. G., Treumann, R. A., Tsagas, C. G., \& Widrow, L. M. 2012, SSRv, 166, 1

Schure, K. M., Bell, A. R., Drury, L. O’C., \& Bykov, A. M. 2012, SSRv, 173,491

Simionescu, A., Werner, N., Urban, O., et al. 2013, ApJ, 775, 4

Skillman, S. W., Hallman, E. J., O'Shea, B. W., et al. 2011, ApJ, 735, 96

Skillman, S. W., O’Shea, B. W., Hallman, E. J., Burns, J. O., \& Norman, M. L. 2008, ApJ, 689, 1063
Skillman, S. W., Xu, H., Hallman, E. J., et al. 2013, ApJ, 765, 21

van Weeren, R. J., Röttgering, H. J. A., Brüggen, M., \& Cohen, A. 2009, A\&A, 508,75

van Weeren, R. J., Röttgering, H. J. A., Brüggen, M., \& Hoeft, M. 2010, Sci, 330,347

van Weeren, R. J., Röttgering, H. J. A., Intema, H. T., et al. 2012, A\&A, 546, A124

Vazza, F., Brüggen, M., Gheller, C., \& Brunetti, G. 2012, MNRAS, 421, 3375

Vazza, F., Brunetti, G., \& Gheller, C. 2009a, MNRAS, 395, 1333

Vazza, F., Brunetti, G., Kritsuk, A., et al. 2009b, A\&A, 504, 33

Vazza, F., Dolag, K., Ryu, D., et al. 2011, MNRAS, 418, 960

Vikhlinin, A., Kravtsov, A., Forman, W., et al. 2006, ApJ, 640, 691

Voit, G. M., Kay, S. T., \& Bryan, G. L. 2005, MNRAS, 364, 909

Walker, S. A., Fabian, A. C., Sanders, J. S., \& George, M. R. 2012, MNRAS, 427, L45 